\section{Safe Handling of Embedding Media}

E. Ann Ellis,

Texas A \& M University

eann.ellis@worldnet.att.net

A previous article documented the health hazards associated with embedding media (1). This article will present specific measures that can be taken to diminish and/or eliminate problems when handling embedding media. Use of personal protective equipment is essential in all laboratory activities and especially when working with embedding media.

It is sometimes better to use alternative chemicals rather than traditional reagents. Propylene oxide, the smallest epoxide, has been used extensively as a transitional solvent with epoxy resins; however, acetonitrile has been used as an alternative reagent. Ethanol or methanol must be substituted for propylene oxide when working with cell cultures in situ and can be substituted for propylene oxide with Spurr's low viscosity resin.

Precautious for handling epoxy embedding media apply equally well to acrylic, polyester and other media. A number of publications provided a background for the following list of precautions (2-6). One thing must be kept in mind when handling dehydrating agents, transitional solvents and embedding media. The same thing that a reagent does to a piece of tissue will be done to your hands, skin, eyes and mucous membranes. In addition, after the tissue is dehydrated, its barrier properties are no longer effective.

1. Carry out all'procedures (dispensing, mixing, infiltration changes, embedding) in a properly operating chemical fume hood. If it is not practical to vent the embedding oven into the hood, precautions should be taken to minimize volatile fumes from the polymerizing resins. Put tops on BEEM and gelatin capsules, and place flat embedding molds in closed petri dishes.

2. Prepare and use embedding mixtures with disposable labware. Transfer individual components, infiltrating and embedding mixtures with disposable glass or plastic pipettes or syringes. Dispose of waste media and utensils in an appropriate manner. Waste transitional solvents and infiltrating mixtures should be put into appropriately labeled containers in the hood. Epoxy resin wastes should not be mixed with acrylic or polyester wastes. Pure resins should be disposed of by polymerization; the polymerized material can then be safely thrown into the trash. Unpolymerized acrylics are not the only danger. Sometimes the surface of acrylic blocks is sticky to touch. This stickiness can be reduced or eliminated by bubbling dry nitrogen or argon gas through the liquid medium before embedding; dry nitrogen gas displaces oxygen, which inhibits polymerization of acrylics. Amine accelerators probably present the greatest hazard of all embedding components, in that amines are strong irritants and extremely volatile (2). Amines should be handled only in a properly functioning hood and dispensed with disposable pipettes or plastic syringes. The used pipette or syringe should be disposed of in a sealed container in the hood, not thrown into the trashcan where people in the lab will be exposed to the vapors of these volatile compounds.

3. Use appropriate gloves as protection against accidental contamination $(4,7)$. Over the years, latex gloves have been used extensively in microscopy laboratories as the only method of hand protection. However, we now know that latex gloves are a poor form of hand protection when handling various embedding media. Orthopedic surgeons demonstrated the necessity of using non-latex gloves in handling acrylics (7). Ringo et al. (4) demonstrated penetration of latex gloves by a number of epoxy resins, especially the diluents such as dibutyl phthalate. Butyl and nitrile rubber gloves have been demonstrated to be resistant to dibutyl phthalate (9). In addition, many people have developed an allergy to latex. Some hospitals and laboratories have dealt with the problem of latex allergy by stopping the use of latex gloves and replacing them with vinyl or nitrile gloves. Barrier creams can be used as additional protection in conjunction with the appropriate gloves for the embedding medium.

Latex gloves are penetrated rapidly by acrylics and exposure results in a painful contact dermatitis in susceptible individuals. Renard et al. (8) reported that butyl rubber and nitrile rubber were more effective barriers to permeation by multifunctional acrylic compounds than natural (latex) rubber. Multifunctional acrylics penetrate glove materials at much lower rates than simple acrylic compounds. It is important that students and technical staff know which resins/embedding media are acrylic and/or methacrylate resins. The following is a list of acrylic and/or methacrylate resins which are used to embed specimens: glycol methacrylate, hydroxypropyl methacrylate, methyl methacrylate/butyl methacrylate, JB-4, Lowicryls (K4M, K11M, HM20, HM23), LR White, LR Gold, Unicryl, Histocryl, Microbed, Immuno-Bed, Osteo-Bed. Acetone is sometimes used as a dehydrating agent and transitional solvent. Nitrile gloves are not resistant to acetone (9) and acetone interferes with the polymerization of LR White and Gold resins (10). An alternative dehydrating agent or transitional solvent should be used in this situation or butyl rubber gloves should be used.

Just as there are no universal solvents, fixatives, or embedding media, there are no absolute choices of disposable gloves or other personal protective devices. We have information to help us choose the appropriate materials and it is our responsibility to acquire and use these devices in an appropriate manner. In addition, we must continually try to educate ourselves and others as new information becomes available.

\section{REFERENCES:}

1. Ellis, E. A. 2003. Embedding media health hazards and medical documentation. Microscopy Today, 11(2):46-47.

2. Bourne, L. B., et al., 1959. Health problems of epoxy resins and amine-curing agents. British Journal of Industrial Medicine, 16:8197.

3. Causton, B. E., et al., 1981. Resins: Toxicity, hazards, and safe handling. Proceedings of Royal Microscopical Society, 16:265-268.

4. Ringo, D. L. et al., 1984. Glove materials for handling epoxy resins. J. Electron Microscopy Technique, 1:417-418.

5. Ellis, E. A. 1989. Embedding media: An overview of hazards and safe handling. EMSA Bulletin, 19(1):83-87.

6. Glauert, A. M. and Day, P. R. 1998. Biological Specimen Preparation for Transmission Electron Microscopy. Princeton University Press, Princeton, NJ.

7. Wagemaekers, T. H. J. M., et al., 1983. Permeability of surgeons' gloves to methyl methacrylates. Acta Orthopaedica Scandinavica, 54:790-795.

8. Renard, E. P et al. 1992. Permeation of multifunctional acrylates through selected protective glove materials. American Industrial Hygiene Association Journal, 53(2):117-123.

9. Lab Safety Supply. 2003. Chemical Compatibility Guide. pg 259. 10. London Resin Company Data Sheets for LR White and LR Gold. 